\title{
EXTENDING CONTINUOUS LINEAR FUNCTIONALS IN CONVERGENCE INDUCTIVE LIMIT SPACES
}

\author{
S. K. KRANZLER AND T. S. McDERMOTT
}

ABstract. Let $E_{n}$ be an increasing sequence of locally convex linear topological spaces such that the dual $E_{n}^{\prime}$ of each has a Fréchet topology (not necessarily compatible with the dual system $\left(E_{n}^{\prime}, E_{n}\right)$ ) weaker than the Mackey topology. Let $E=\bigcup_{n=1}^{\infty} E_{n}$, $F$ be a subspace of $E$ and $\tau$ the inductive limit convergence structure on $E$. Conditions are given which insure that every $\tau$ continuous linear functional on $F$ has a $\tau$-continuous linear extension to $E$. This result generalizes a theorem of $\mathrm{C}$. Foias and G. Marinescu.

Let $(E, \tau)$ be a convergence vector space and $f$ a continuous linear functional defined on a subspace $F$ of $E$. There are relatively few situations where it is known whether $f$ has a continuous linear extension to $E$. In [3] C. Foias and G. Marinescu showed that, in inductive limits of reflexive Banach spaces, sequentially continuous linear functionals defined on sequentially closed subspaces have continuous linear extensions to the whole space. In the terminology of [2] this theorem yields, "If $(E, \tau)$ is a convergence inductive limit of a sequence of reflexive Banach spaces, then every $\tau$-continuous linear functional on a $\tau$-closed subspace of $E$ has a $\tau$-continuous linear extension to $E$." It is the purpose of this note to adapt the method of proof used in [3] to prove a more general result (Theorem 1). M. De Wilde [1] has extended the Foias-Marinescu theorem in a different direction to inductive limits of Banach spaces in which the canonical injections are weakly compact, relieving the reflexivity condition. This line of thought has been carried over to an extension theorem in the context of spaces with "boundedness structures" (espaces bornologiques) by $\mathrm{H}$. Hogbe-Nlend [4, p. 66].

THEOREM 1. Let $E_{1} \subseteq E_{2} \subseteq E_{3} \subseteq \cdots$ be a sequence of locally convex linear topological spaces such that for each $n$

(1) the canonical injection $i_{n}: E_{n} \rightarrow E_{n+1}$ is continuous,

(2) there is a Fréchet topology $T_{n}$ on $E_{n}^{\prime}$ (not necessarily compatible with the dual system $\left.\left(E_{n}^{\prime}, E_{n}\right)\right)$ weaker than the Mackey topology $\tau\left(E_{n}^{\prime}, E_{n}\right)$, and

(3) the transpose maps $i_{n}^{*}: E_{n+1}^{\prime} \rightarrow E_{n}^{\prime}$ are continuous for the topologies $T_{n+1}$ and $T_{n}$.

Received by the editors December 1, 1972 and, in revised form, July 12, 1973. AMS (MOS) subject classifications (1970). Primary 46A05, 46A20.

(c) American Mathematical Society 1974 
Let $F$ be a linear subspace of $E=\bigcup_{n=1}^{\infty} E_{n}$, such that for each $n, F_{n}$ is closed in $E_{n}$, where $F_{n}=E_{n} \cap F$. If $\varphi$ is a linear functional on $F$ such that for each $n, \varphi_{n}=\left.\varphi\right|_{F_{n}}$ is continuous, then there exists a linear functional $\hat{\varphi}$ on $E$ such that $\left.\hat{\varphi}\right|_{F}=\varphi$ and $\left.\hat{\varphi}\right|_{E_{n}}$ is continuous for each $n$.

The proof requires the following lemmas.

Lemma 1. Let $E$ and $F$ be two locally convex linear topological spaces, and $J: E \rightarrow F$ a continuous linear mapping. Let $M$ be a subspace of $E$ and $N$ a closed subspace of $F$ such that $J^{-1}(N) \subseteq M$. Then $J^{*}\left[N^{\circ}\right]$ is $\sigma\left(E^{\prime}, E\right)$ dense in $M^{\circ}$.

Proof. It suffices to show that $\left[J^{*}\left(N^{\circ}\right)\right]^{\circ \circ} \supseteq M^{\circ}$. This would follow if $M \supseteq\left[J^{*}\left(N^{\circ}\right)\right]^{\circ}$. But if $z \in\left[J^{*}\left(N^{\circ}\right)\right]^{\circ}$, then for all $y \in J^{*}\left(N^{\circ}\right),\langle z, y\rangle=0$. That is, for all $x \in N^{\circ}, 0=\left\langle z, J^{*}(x)\right\rangle=\langle J(z), x\rangle$, and hence $J(z) \in N^{\circ \circ}=N$. But, then, $z \in J^{-1}(N) \subseteq M$.

LEMMA 2. Under the hypotheses of the theorem, for each $n$ there is a family $\rho_{n, k}$ of seminorms defining the topology $T_{n}$ such that

(1) $\rho_{n, k}(z) \leqq \rho_{n, k+1}(z) \quad \forall k, \forall n$, and

(2) $\rho_{n, k}\left(i_{n}^{*}(z)\right) \leqq \rho_{n+1, k}(z) \quad \forall k, \forall n$.

Proof. Choose $\rho_{1, k}$ on $E_{1}^{\prime}$ satisfying (1) and defining the topology $T_{1}$. Since $i_{1}^{*}$ is continuous with respect to $T_{2}$ and $T_{1}$, if $V_{m}(0)$ is a decreasing base of absolutely convex neighborhoods for $E_{2}^{\prime}$, there is a subsequence $V_{m_{k}}(0)$ such that

$$
\rho_{1, k}\left(i_{1}^{*}(z)\right) \leqq 1 \quad \text { for all } z \in V_{m_{k}}
$$

and $V_{m_{k}} \subset V_{m_{k-1}}$. Let $\rho_{2, k}$ be the seminorm determined by $V_{m_{k}}$. Then

$$
\rho_{2, k}(z) \leqq \rho_{2, k+1}(z) \text {, and } \rho_{1, k}\left(i_{1}^{*}(z)\right) \leqq \rho_{2, k}(z) \text {. }
$$

It is clear that this process may be repeated by induction so as to yield the conclusion of the lemma.

ProOF OF THEOREM 1. For each $n$, let $\rho_{n, k}$ be a sequence of seminorms defining the topology $T_{n}$ for $E_{n}^{\prime}$ satisfying (1) and (2) in Lemma 2. By Lemma $1, i_{n}^{*}\left(F_{n+1}^{\circ}\right)$ is $\sigma\left(E_{n}^{\prime}, E_{n}\right)$-dense in $F_{n}^{\circ}$, hence $\tau\left(E_{n}^{\prime}, E_{n}\right)$-dense in $F_{n}^{\circ}$, and therefore $T_{n}$-dense in $F_{n}^{\circ}$. For each $n$, let $f_{n}$ be a continuous extension of $\varphi_{n}$ to $E_{n}$. Setting $z_{1}=0$ and using the $T_{n}$-denseness of $i_{n}^{*}\left(F_{n+1}^{\circ}\right)$ in $F_{n}^{\circ}$, we may construct a sequence $z_{n}$ such that

$$
\begin{gathered}
z_{n} \in F_{n}^{\circ}, \\
\rho_{n-1, n-1}\left(f_{n-1}+z_{n-1}-i_{n}^{*}\left(f_{n}+z_{n}\right)\right)<(1 / 2)^{n-1} .
\end{gathered}
$$




\section{Setting}

$$
\delta_{n}=f_{n}+z_{n}-i_{n}^{*}\left(f_{n+1}+z_{n+1}\right)
$$

let

$$
g_{n}=f_{n}+z_{n}-\delta_{n}-i_{n}^{*}\left(\delta_{n+1}\right)-i_{n}^{*} \circ i_{n+1}^{*}\left(\delta_{n+2}\right)-\cdots .
$$

The series for $g_{n}$ converges in $E_{n}^{\prime}$ since given $\rho_{n, m}$,

$$
\begin{aligned}
& \rho_{n, m}\left(i_{n}^{*} \circ \cdots \circ i_{n+r}^{*}\left(\delta_{n+r+1}\right)\right) \leqq \rho_{n+1, m}\left(i_{n+1}^{*} \circ \cdots \circ i_{n+r}^{*}\left(\delta_{n+r+1}\right)\right) \\
& \leqq \cdots \leqq \rho_{n+r, m}\left(i_{n+r}^{*}\left(\delta_{n+r+1}\right)\right) \leqq \rho_{n+r+1, m}\left(\delta_{n+r+1}\right) \\
& \leqq \rho_{n+r+1, n+r+1}\left(\delta_{n+r+1}\right) \leqq \frac{1}{2^{n+r+1}} \text { if } m \leqq n+r+1 .
\end{aligned}
$$

Hence, the sequence of partial sums is Cauchy, and since $\left(E_{n}^{\prime}, T_{n}\right)$ is complete, $g_{n} \in E_{n}^{\prime}$ is defined. Define $\hat{\varphi}(x)=g_{n}(x), x \in E_{n}$. Then $\hat{\varphi}$ is a well-defined linear functional on $E$ since, by (3),

$$
\begin{aligned}
i_{n}^{*}\left(g_{n+1}\right) & =i_{n}^{*}\left(f_{n+1}\right)+i_{n}^{*}\left(z_{n+1}\right)-i_{n}^{*}\left(\delta_{n+1}\right)-i_{n}^{*} \circ i_{n+1}^{*}\left(\delta_{n+2}\right)-\cdots \\
& =f_{n}+z_{n}-\delta_{n}-i_{n}^{*}\left(\delta_{n+1}\right)-i_{n}^{*} \circ i_{n+1}^{*}\left(\delta_{n+2}\right)-\cdots \\
& =g_{n} .
\end{aligned}
$$

Moreover, $\left.\hat{\varphi}\right|_{E_{n}}=g_{n}$ and hence is continuous, while $\left.\hat{\varphi}\right|_{F_{n}}=\left.g_{n}\right|_{F_{n}}=$ $\left.f_{n}\right|_{F_{n}}=\varphi_{n}$. Hence $\left.\hat{\varphi}\right|_{F}=\varphi$.

REMARK 1. One may readily find examples to which Theorem 1 applies through the following observation. Let $E_{n}$ be an increasing sequence of locally convex spaces such that for each $n$, there is a Fréchet space $G_{n}$ with topology $T_{n}$ and $G_{n}^{\#} \supseteq E_{n} \supseteq G_{n}^{\prime}$, where ${ }^{\#}$ and ' indicate the algebraic and continuous duals respectively. If $E_{n}$ is endowed with any topology $S_{n}$ of the dual pair $\left(E_{n}, G_{n}\right)$, then $E_{n}^{\prime}=G_{n}$ and $\tau\left(E_{n}^{\prime}, E_{n}\right) \geqq T_{n}$. Let the canonical injections $i_{n}: E_{n} \rightarrow E_{n+1}$ be $S_{n}, S_{n+1}$ continuous and suppose $i_{n}\left(G_{n}^{\prime}\right) \subset G_{n+1}^{\prime}$ (this last condition is automatically satisfied if $\left.E_{n}=G_{n}^{\prime}\right)$. Since $\left.\sigma\left(E_{n}, E_{n}^{\prime}\right)\right|_{G_{n}^{\prime}}=\sigma\left(G_{n}^{\prime}, G_{n}\right),\left.i_{n}\right|_{G_{n}^{\prime}}$ is continuous with respect to the topologies $\sigma\left(G_{n}^{\prime}, G_{n}\right)$ and $\sigma\left(G_{n+1}^{\prime}, G_{n+1}\right)$. Thus $i_{n}^{*}: G_{n+1} \rightarrow G_{n}$ is continuous with respect to $\tau\left(G_{n+1}, G_{n+1}^{\prime}\right)$ and $\tau\left(G_{n}, G_{n}^{\prime}\right)$, that is, with respect to $T_{n+1}$ and $T_{n}$. We see then that (2) and (3) of Theorem 1 are both satisfied in this context.

We now give a specific illustration of the situation described in Remark 1.

Proposition 1. Let $F_{n}$ be a sequence of Fréchet spaces, and for each $n$ let $F_{n}^{\prime}$ have a locally convex topology compatible with the pairing $\left(F_{n}^{\prime}, F_{n}\right)$. Let $E_{n}=\prod_{k=1}^{n} F_{n}^{\prime}$ be endowed with the product topology $S_{n}$ and let $E=$ $\bigcup_{n=1}^{\infty} E_{n}$ be the inductive limit of the sequence $E_{n}$ (i.e. $E$ is the locally 
convex sum of the $\left.F_{n}^{\prime}\right)$. Suppose $M$ is a subspace of $E$ such that for all $n$, $M_{n}=M \cap E_{n}$ is closed in $E_{n}$. If $\varphi$ is a linear functional on $M$ such that for all $n,\left.\varphi\right|_{M_{n}}$ is continuous, then $\varphi$ has a continuous linear extension to $E$.

Proof. Set $G_{n}=\prod_{k=1}^{n} F_{k}$ and apply the reasoning of Remark 1 .

REMARK 2. The theorem yields no new information in the case that the $E_{n}$ are Banach spaces. This follows by the fact that in $E_{n}^{\prime}$ any Fréchet topology $T_{n}$ weaker than $\tau\left(E_{n}^{\prime}, E_{n}\right)$ is weaker than $\beta\left(E_{n}^{\prime}, E_{n}\right)$, the (complete) norm topology. Hence, by the Baire Category Theorem $T_{n}=$ $\beta\left(E_{n}^{\prime}, E_{n}\right)=\tau\left(E_{n}^{\prime}, E_{n}\right)$, and so $E_{n}$ is reflexive.

\section{REFERENCES}

1. M. DeWilde, Sur un type particulier de limite inductive, Bull. Soc. Roy. Sci. Liège 35 (1966), 545-551. MR 35 \#5890.

2. H. R. Fischer, Limesräume, Math. Ann. 137 (1959), 269-303. MR 22 \#225.

3. C. Foias and G. Marinescu, Fonctionelles linéaires dans les réunions dénombrables d'espaces de Banach réflexifs, C. R. Acad. Sci. Paris 261 (1965), 4958-4960. MR 33 \#3095.

4. H. Hogbe-Nlend, Théorie des bornologies et applications, Lecture Notes in Math., Springer-Verlag, Berlin, 1971.

5. John Horvàth, Topological vector spaces and distributions. Vol. I, AddisonWesley, Reading, Mass., 1966. MR 34 \#4863.

Department of Mathematics, University of Hawail, Honolulu, Hawail 96822 